\title{
Psychological factors are important to return to pre-injury sport activity after anterior cruciate ligament reconstruction: expect and motivate to satisfy
}

\author{
Sofi Sonesson $^{1} \cdot$ Joanna Kvist $^{1} \cdot$ Clare Ardern $^{1,2,3}$ • Annika Österberg ${ }^{1,4}$. \\ Karin Grävare Silbernagel ${ }^{5}$
}

Received: 4 February 2016 / Accepted: 12 August 2016 / Published online: 25 August 2016

(C) The Author(s) 2016. This article is published with open access at Springerlink.com

\begin{abstract}
Purpose To describe individuals' expectations, motivation, and satisfaction before, during, and after rehabilitation for ACL reconstruction and to explore how these factors were associated with return to pre-injury sport activity at 1 -year follow-up.

Methods Sixty-five individuals (34 males), median age 22 (15-45) years, scheduled for ACL reconstruction participated. Participants completed the International Knee Documentation Committee Subjective Knee Form (IKDC-SKF) and questions about expectations, satisfaction, and motivation pre-operatively and at 16 and 52 weeks after surgery.

Results Prior to surgery, $86 \%$ of participants stated that their goal was to return to their pre-injury sport activity. Those who had returned to their pre-injury sport activity at 52 weeks were more motivated during rehabilitation to return to their pre-injury activity level, more satisfied with their activity level and knee function at 52 weeks, and scored significantly higher on the IKDC-SKF [median 92.0 (range 66.7-100.0)] at 52 weeks, compared to those who had not returned [median 77.6 (range 50.6-97.7)].
\end{abstract}

Sofi Sonesson

Sofi.sonesson@liu.se

1 Division of Physiotherapy, Linköping University, 581 83 Linköping, Sweden

2 School of Allied Health, Faculty of Health Sciences, La Trobe University, Melbourne, VIC, Australia

3 Aspetar Orthopaedic and Sports Medicine Hospital, Doha, Qatar

4 Centre for Clinical Research Sörmland, Uppsala University, Eskilstuna, Sweden

5 Department of Physical Therapy, University of Delaware, Newark, DE, USA
Conclusion Prior to ACL reconstruction, most participants expected to return to their pre-injury activity level. Higher motivation during rehabilitation was associated with returning to the pre-injury sport activity. The participants who had returned to their pre-injury sport activity were more satisfied with their activity level and knee function 1 year after the ACL reconstruction. Facilitating motivation might be important to support individuals in achieving their participation goals after ACL reconstruction.

Level of evidence Prospective cohort study, Level II.

Keywords ACL $\cdot$ Return to sport · Patient-reported outcome $\cdot$ Patient perspective $\cdot$ Psychology $\cdot$ Activity level

\section{Introduction}

Anterior cruciate ligament (ACL) injuries are common in young sports-active people (15-30 years). The injury leads to altered knee joint biomechanics and functional instability, and may require surgical treatment [9]. One of the most important reasons for performing ACL reconstruction surgery is to reduce knee instability in order to facilitate a return to the pre-injury physical activity level $[8,15,18]$.

Patients have high expectations of recovery after ACL reconstruction; the majority expect good knee function and a return to the pre-injury level of sport [20]. However, these high expectations may not be fulfilled. Many athletes do not return to their pre-injury level of sports even though they are physically rehabilitated $[19,26,27]$ and despite the fact that the goal of reconstruction and rehabilitation is to return to the preinjury level [42]. Most patients (85-90 \%) report good knee function after ACL reconstruction, but less than half return to their pre-injury competitive sport [7]. Unfulfilled expectations might also be associated with low satisfaction [13, 37]. 
Many factors influence whether an individual returns to sport $[14,18]$. However, it is not fully understood what factors are associated with successful rehabilitation, resulting in a return to sports, and patient satisfaction after ACL reconstruction. Good physical function is a prerequisite to return to sport $[1,32,40]$, and adequate post-operative rehabilitation is fundamental to facilitate recovery of the physical capacity required to participate in sport activity [15]. In addition, psychological factors and their impact on recovery, rehabilitation, and return to sport have received more attention in recent years $[2,5,12,14,16,18,21,28$, $33,34,39]$. Impaired mood has a negative impact on ACL rehabilitation [31], and in line with this, high optimism is associated with better knee function after rehabilitation [38]. High motivation also seems to be of importance for returning to sport after an ACL reconstruction [17, 21].

Rehabilitation is a long and demanding process that patients are often not fully mentally prepared for [23]. Data on patients' expectations and motivation before an ACL reconstruction and during rehabilitation can provide enhanced knowledge of how these factors influence satisfaction and return to sport. Therefore, the purposes of the present study were to describe individuals' expectations, motivation, and satisfaction before, during and after rehabilitation for ACL reconstruction and to explore how these factors were associated with return to pre-injury sport activity at 1-year follow-up. The hypotheses were that preoperative expectations would be high and motivation would be important in order to return to pre-injury sport activity. Increased knowledge of these psychological factors can contribute to improve the current treatment protocols for this patient population.

\section{Materials and methods}

For this prospective cohort study, all patients with an ACL injury, who were scheduled for an ACL reconstruction between January 2012 and June 2013 at one of three orthopaedic clinics in Sweden, were invited to participate. The inclusion criteria were: unilateral ACL injury; first time ACL reconstruction; and age 15-45 years at the time of ACL reconstruction. The exclusion criteria were: previous ACL reconstruction (to either knee); other major injuries to either knee (i.e. grade III collateral ligament injury, PCL injury, or grade III articular cartilage injury). Of 161 eligible patients, 65 patients completed the pre-operative questionnaire.

\section{Treatment}

All patients received a hamstring graft for ACL reconstruction. Patients were treated according to standard Swedish clinical guidelines. According to the treatment algorithm, patients with ACL injury completed a period of structured rehabilitation (typically around 3 months) before a decision was made regarding surgery. Patients were advised to have ACL reconstruction if they continued to experience functional instability with recurrent giving way episodes or they wished to return to contact or pivoting sport or employment with high demands of knee function. Pre-operative information about the surgery, the healing process, the rehabilitation, and knee function after surgery should be provided to all patients before ACL reconstruction. The pre-operative information included rehabilitation time frames.

\section{Questionnaires}

Electronic versions of a study-specific questionnaire (Table 1) and the Swedish International Knee Documentation Committee Subjective Knee Form (IKDC-SKF) [24] were sent to all participants via email before the scheduled ACL reconstruction, and at 16 and 52 weeks post-operatively (participants who did not have an email address were sent paper copies of the questionnaires). For each assessment, up to two reminders were sent. The assessments were timed to capture participants' responses at clinically relevant times.

The study-specific questionnaire included questions about expectations, motivation to return to pre-injury activity level, and satisfaction (Table 1). The IKDC-SKF is a knee-specific questionnaire that evaluates symptoms, function, and sport activity. The instrument contains 10 items. The total score ranges $0-100$ where 100 represents no limitations [24]. The IKDC-SKF has been translated and culturally adapted to Swedish (personal communication; manuscript under review).

\section{Ethical approval}

Ethical approval was obtained from the Ethics Committee at Linköping University (Dnr 2011/450-31). All patients provided written informed consent prior to participation.

\section{Statistical analyses}

Analyses were performed using the Statistical Package for the Social Sciences (SPSS) version 21 (IBM Corp, Armonk, New York, USA). Descriptive statistics were calculated for all outcomes. Mann-Whitney $U$ tests and Pearson Chi-square tests were used to compare responses from participants who had returned to their pre-injury sport activity at the 52-week follow-up to those who had not returned. Significance was set at $P<0.05$ for all analyses. Since this is a prospective exploratory study that for the first time describes individual's expectation, motivation 


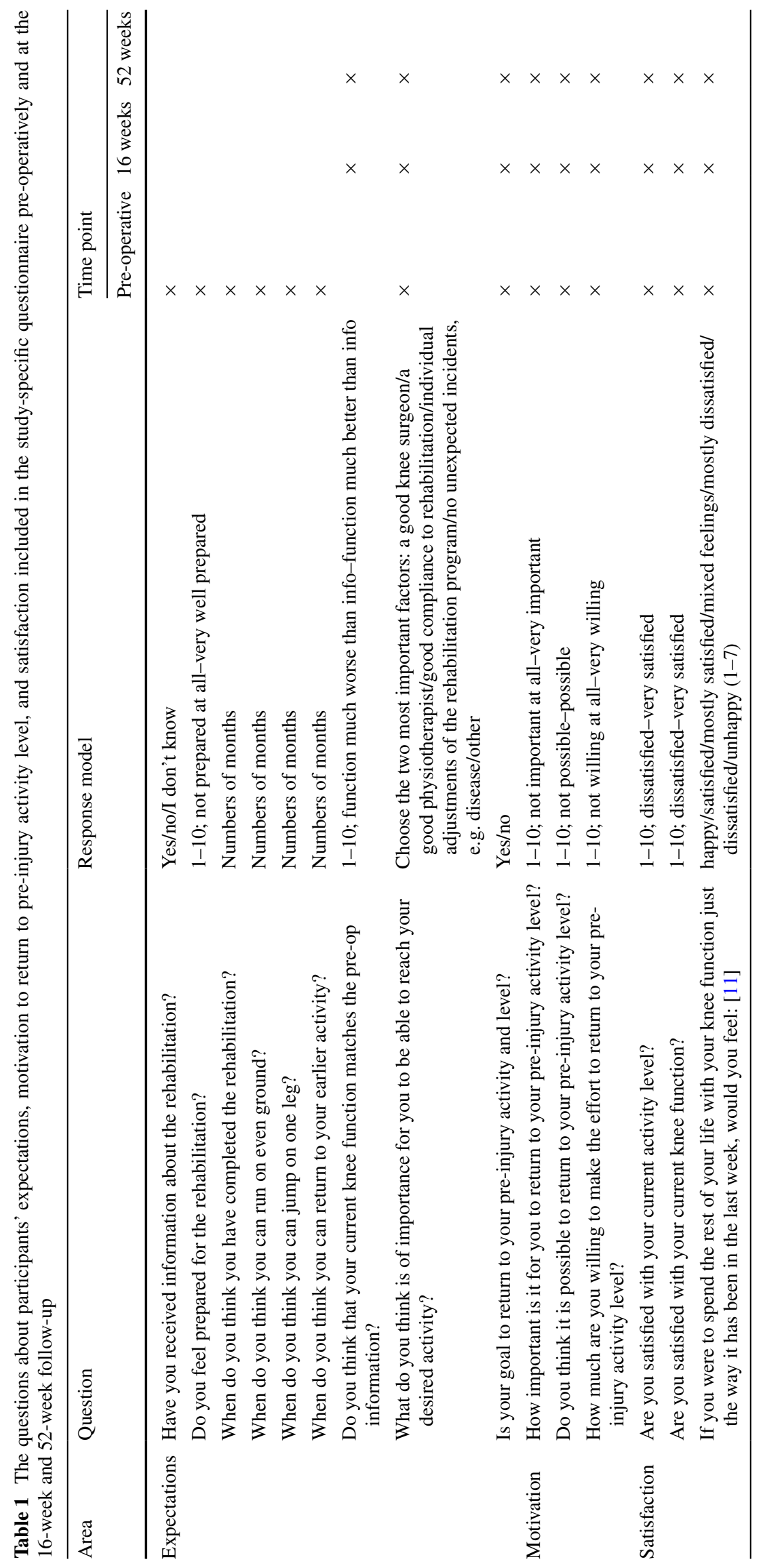


and satisfaction before, during and after ACL reconstruction, there were no known data to use for a power calculation. The post hoc evaluation revealed that the power of the study was 0.82 (one-tailed) for the question "Do you think it is possible to return to your pre-injury activity level?" at 52 weeks.

\section{Results}

Questionnaires were sent to 161 patients before ACL reconstruction; 65 participants completed the pre-operative questionnaire at a median of 1 week pre-operatively and were included in the follow-up. Of these, 46 participants completed the 16-week questionnaire, and 43 participants completed the questionnaire at 52-week follow-up. The participants were a median 22 (range 15-45) years old at the time of ACL reconstruction. There were 34 males (52\%) and 31 females $(48 \%)$ (Table 2). Time from injury to surgery was a median $9(0-128)$ months (Table 2$)$.

\section{Pre-operative expectations}

All except two participants reported that they received preoperative information regarding surgery and rehabilitation. Most indicated that pre-operatively they felt prepared for rehabilitation (61 participants (95\%) estimated 6-10). Nine participants $(14 \%)$ estimated their rehabilitation would take 5-6 months to complete, 48 participants (76\%) estimated 7-12 months, and 6 participants (10\%) estimated more than 12 months rehabilitation duration. There were no differences between participants who had returned to their pre-injury sport activity at 52 weeks and those who had not returned, regarding the pre-operative estimated time to completion of rehabilitation, estimated time to be able to run/jump, or estimated time to return to pre-injury sport activity (n.s.).

At the 16- and 52-week follow-ups, participants estimated their current knee function slightly better compared to the pre-operative information about approximate time frames for achievement of rehabilitation goals (median 6, range $1-10$ and median 7 , range $3-10$ ).

Participants stated that good compliance to rehabilitation, a good knee surgeon, and a good physiotherapist were the most important factors in order to be able to reach their desired sport activity (Table 3 ). There were no differences between participants who had returned to their pre-injury sport activity at 52 weeks compared to participants who had not returned regarding what factors they estimated were of importance (n.s.).

Pre-operatively, 54 of 63 participants $(86 \%)$ stated that their goal was to return to their pre-injury sport activity, and at 16 weeks, 35 of 46 participants (76\%) had that same goal. Returning to the pre-injury sport activity at 52 weeks was not related to whether participants said their goal was to return at either the pre-operative or 16-week follow-up (n.s.). Satisfaction with knee function (1-7 scale; Table 1) at 52 weeks was not related to participants' pre-operative goal for returning to sport activity (n.s.).

\section{Motivation}

For most participants, it was very important to return to their pre-injury activity level (Table 4).

Participants who had returned to their pre-injury sport activity at 52 weeks, to a greater extent estimated pre-operatively that it was possible to return to their earlier activity level compared to those who had not returned at 52 weeks $(P=0.019$, Table 4). Those who had returned to their pre-injury sport activity at 52 weeks were more motivated during rehabilitation to return to their earlier activity level compared to those who had not returned to their pre-injury sport activity (Table 4).

\section{Satisfaction}

Satisfaction increased during rehabilitation. At 52 weeks, most participants were satisfied with their current activity level (median 7.5, range 1-10) and current knee function (median 8, range 1-10) (Table 4). Participants who had returned to their pre-injury sport activity at 52 weeks were more satisfied with their activity level $(P=0.002)$ and knee function $(P=0.009)$ at 52 weeks compared to those who had not returned (Table 4).

\section{Discussion}

The main finding in the present study was that participants who had returned to their pre-injury sport activity at 1 year after surgery were more motivated during rehabilitation to return to their earlier activity level, compared to participants who had not returned to their pre-injury sport activity. Further, those who had returned to their pre-injury sport activity at 52 weeks were more satisfied with their current activity level and knee function. Prior to surgery, most participants stated that their goal was to return to their preinjury sport activity and for the majority, this was of great importance. Consequently, motivation seems to be a key issue in ACL rehabilitation in order to reach a high activity level and satisfaction. Motivation is probably a prerequisite in order to achieve good adherence to the rehabilitation regime during recovery $[10,29,35]$.

Since the standard practice is that the decision for ACL reconstruction is based on functional knee instability, the desire to return to contact or pivoting sport, or a knee 


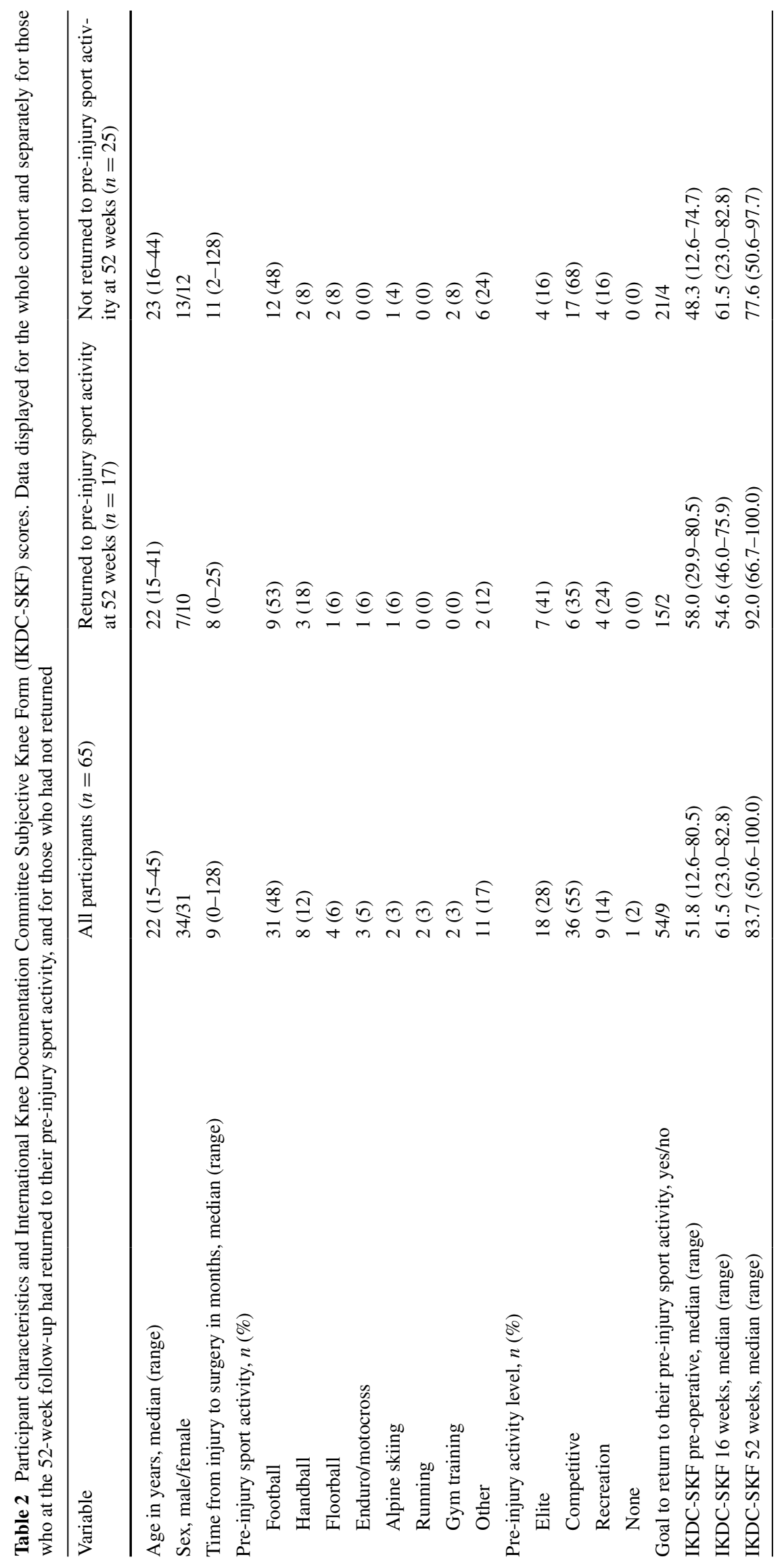


Table 3 Perceptions of what factors are of importance in order to be able to reach desired sport activity, pre-operatively and at follow-up. Participants chose the two most important factors

\begin{tabular}{|c|c|c|c|}
\hline \multirow[t]{2}{*}{ Factor } & \multicolumn{3}{|c|}{$\begin{array}{l}\text { Number }(\%) \text { who estimated that the factor was of importance in order } \\
\text { to be able to reach their desired sport activity }\end{array}$} \\
\hline & Pre-operative & 16 weeks & 52 weeks \\
\hline A good knee surgeon & $38(59.4)$ & $16(34.8)$ & $13(31.0)$ \\
\hline A good physiotherapist & $15(23.4)$ & $26(56.5)$ & $28(66.7)$ \\
\hline Good compliance to rehabilitation & $58(90.6)$ & $43(93.5)$ & $37(88.1)$ \\
\hline Individual adjustments of the rehabilitation program & $14(21.9)$ & $5(10.9)$ & $5(11.9)$ \\
\hline No unexpected incidents, e.g. disease & $7(10.9)$ & $3(6.5)$ & $2(4.8)$ \\
\hline Other & $1(1.6)$ & $1(2.2)$ & $2(4.8)$ \\
\hline
\end{tabular}

demanding job, it is possible that a majority of patients receiving ACL reconstruction were highly motivated to undergo surgery and rehabilitation, and return to sport. Most participants in our study stated before their surgery that it was of great importance to return to their pre-injury activity level. Further, the majority of participants expected to do so. Patients' expectations of recovery after ACL reconstruction are high [20], and expectations are a prognostic factor for physical recovery [30]. The current Swedish treatment model for ACL injury, including detailed pre-operative information, seems to provide adequate information to patients, supporting individuals to establish realistic expectations. In our study, participants' estimations of time to accomplishment of rehabilitation goals and return to pre-injury sport activity were within the approximate time frames specified in the rehabilitation regime. Consequently, it is reasonable to suggest that participants had realistic expectations that reflected current published clinical guidelines and recommendations $[1,8,22,43]$.

Fulfilment of expectations is correlated with improved patient satisfaction [13, 37, 41]. In our study, satisfaction changed over time. Satisfaction with activity level and knee function was low before surgery and increased during rehabilitation. Returning to the pre-injury sport activity was associated with higher satisfaction. Consistent with this, recent research has also demonstrated that returning to physical activity participation is important for people's satisfaction after treatment for ACL injury $[3,36]$. This may be explained by the fact that return to sport is the goal for a majority of the individuals who undergo ACL reconstruction. Satisfaction following anterior cruciate ligament reconstruction is also strongly linked to patients' perceptions of symptoms and function $[25,36]$. Hence, patient satisfaction seems to reflect the outcome of the factors that are of greatest importance to the individual. Consequently, returning to the pre-injury sport activity seems to be of great importance for the individual and is associated with patient satisfaction.

Psychological factors have a critical impact on returning to physical activity following ACL reconstruction $[2,5,12$, $14,16,18,21,28,34,39]$. We hypothesized that patients' motivation during ACL rehabilitation would be essential for optimal outcomes in terms of returning to the pre-injury sport activity. In support of this, female football players who reported higher motivation to return to sports were more likely to return to playing football after ACL reconstruction [17].

Rehabilitation after ACL reconstruction has been acknowledged to be fundamental for the possibility to return to sport [15]. The rehabilitation process is long, and demands great effort from the individual. High motivation may result in good adherence to the rehabilitation protocol, and this might be important for reaching the levels of physical function that is recommended before return to sport [40]. In our study, participants who were more motivated to return to sport achieved that goal to a greater extent, which confirms other research showing a relationship between motivation and return to sport [6]. The clinical application of this finding is that facilitating motivation might be important to support individuals in achieving their participation goals after ACL reconstruction.

Motivation may need more attention in the clinical setting and may need to be addressed more specifically during rehabilitation. Current rehabilitations programs focus mainly on recovering physical function (impairment focus) and not on achieving psychological readiness to return to sport. Both physical readiness and psychological readiness to return to sport are important for returning, but they may not coincide [4]. A rehabilitation program may need to be complemented with a clearer psychological approach to maintain the patient's motivation to return to the pre-injury sport activity. This may have the potential to improve returning to pre-injury sport activity and promote participation in physical activity. However, these hypotheses need to be tested in future research. Assessment of motivation before surgery has the potential to identify need for specific, individualized interventions aimed at improving psychological readiness to return to sport $[12,16]$. Our study supports the need for further research to develop successful strategies to address psychological factors as part of rehabilitation after ACL reconstruction. 


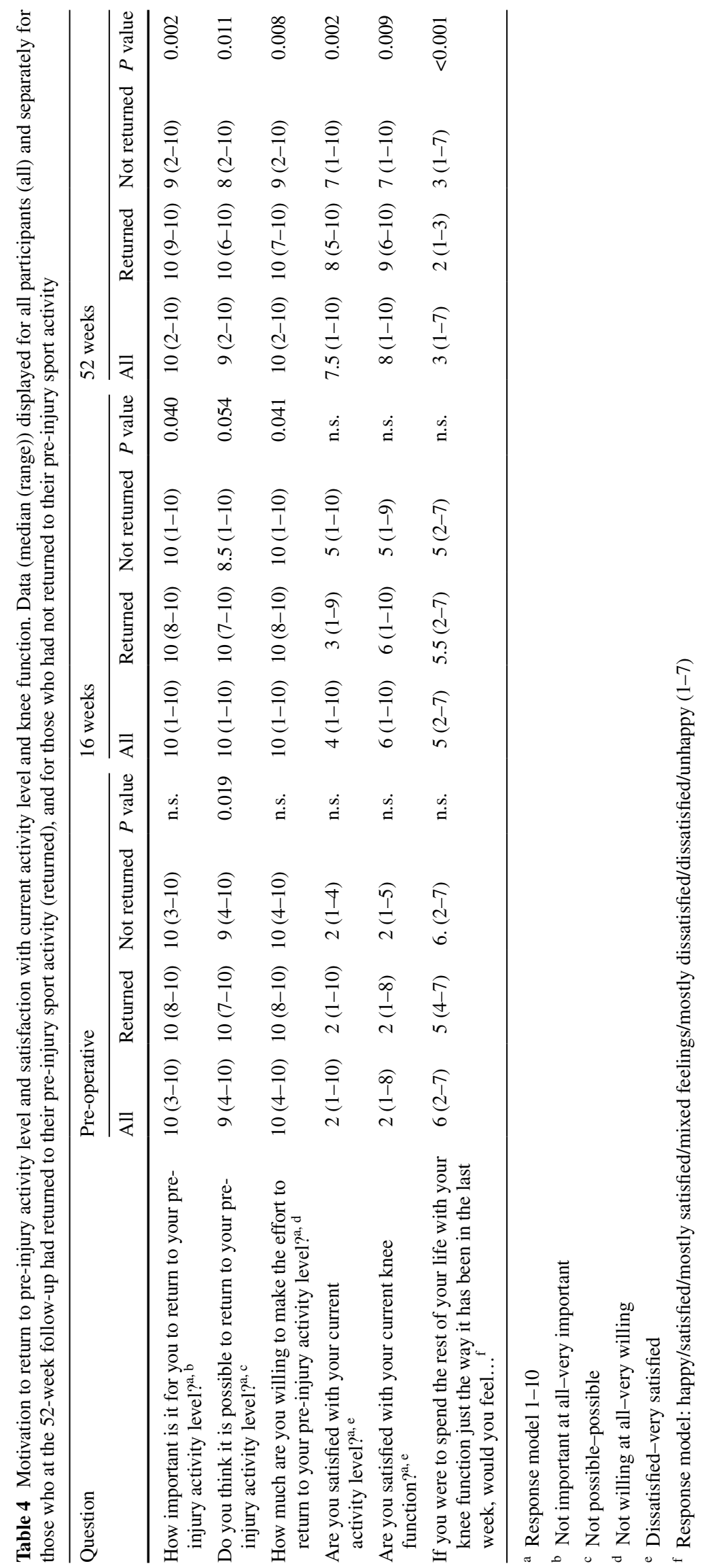


It is important to keep in mind that the participants underwent rehabilitation according to standard practice in Sweden and all except two participants received preoperative information. It is likely that the provision of pre-operative information contributed to the fact that most participants felt prepared for rehabilitation and had realistic expectations. Consequently, accurate pre-operative information seems to be crucial to help patients establish realistic expectations. Individuals who have unrealistic expectations of ACL reconstruction may not be adequately prepared, and may need extra support during rehabilitation to maintain sufficient motivation. Although, since almost all participants in our study received pre-operative information, no comparison could be made with individuals who did not receive pre-operative information.

A strength of our study is that prospective data were collected before surgery, during, and after the rehabilitation period. The objective of the study design was to evaluate participants' responses at clinically relevant times (i.e. immediately prior to the surgical intervention, at the time when sport-specific exercises are typically introduced, and when rehabilitation is expected to have been completed).

A limitation of the present study is the small sample size, and therefore, the results need to be confirmed in future studies. High motivation can be assumed to be a prerequisite to good adherence to a rehabilitation protocol; however, we did not empirically evaluate adherence in our study. Further, there are no data on whether every participant was cleared to return to sport or exactly when participants returned to sport. Instead, participants answered whether they had returned or not to their pre-injury sport activity at 52 weeks after surgery. This raises the potential for recall bias. However, given that returning to sport is a discrete and typically highly anticipated event, the chances of it being influenced by recall bias are likely to be low. Moreover, besides the psychological factors analysed in our study, other factors such as the surgery, rehabilitation and physical function may influence whether an individual returns to sport.

This study found that higher motivation during the rehabilitation was associated with returning to pre-injury sport activity. Facilitating motivation during the rehabilitation might have a beneficial effect on the person's ability to achieve their participation goal.

\section{Conclusion}

The majority of participants expected to return to their pre-injury activity level after ACL reconstruction. Motivation during rehabilitation was associated with returning to the pre-injury sport activity. Participants who had returned to their pre-injury sport activity were more satisfied with their activity level and knee function 1 year after ACL reconstruction compared to those who had not returned to their pre-injury sport activity.

Acknowledgments The authors thank Henrik Magnusson at Linköping University for statistical support. This study was supported by the Faculty of Health Sciences at Linköping University and by grants from the Swedish Centre for Research in Sports.

Authors' contribution SS participated in study design, performed analysis and interpretation of data and drafted the manuscript. JK participated in study design, data collection, analysis and interpretation of data, and manuscript revisions. CA participated in interpretation of data and manuscript revisions. AÖ participated in study design, data collection, and manuscript revisions. KGS participated in study design, interpretation of data, and manuscript revisions. All authors read and approved the final manuscript.

\section{Compliance with ethical standards}

Conflict of interest The authors declare that they have no conflict of interest.

Funding This study was supported by the Faculty of Health Sciences at Linköping University and by grants from the Swedish Centre for Research in Sports.

Ethical approval Ethical approval was obtained from the Ethics Committee at Linköping University (Dnr 2011/450-31).

Informed consent All patients provided written informed consent prior to participation.

Open Access This article is distributed under the terms of the Creative Commons Attribution 4.0 International License (http://creativecommons.org/licenses/by/4.0/), which permits unrestricted use, distribution, and reproduction in any medium, provided you give appropriate credit to the original author(s) and the source, provide a link to the Creative Commons license, and indicate if changes were made.

\section{References}

1. Adams D, Logerstedt DS, Hunter-Giordano A, Axe MJ, SnyderMackler L (2012) Current concepts for anterior cruciate ligament reconstruction: a criterion-based rehabilitation progression. J Orthop Sports Phys Ther 42(7):601-614

2. Ardern CL (2015) Anterior cruciate ligament reconstruction-not exactly a one-way ticket back to the preinjury level: a review of contextual factors affecting return to sport after surgery. Sports Health 7(3):224-230

3. Ardern CL, Osterberg A, Sonesson S, Gauffin H, Webster KE, Kvist J (2016) Satisfaction with knee function after primary anterior cruciate ligament reconstruction is associated with selfefficacy, quality of life, and returning to the preinjury physical activity. Arthroscopy. doi:10.1016/jarthro201601035

4. Ardern CL, Osterberg A, Tagesson S, Gauffin H, Webster KE, Kvist J (2014) The impact of psychological readiness to return to sport and recreational activities after anterior cruciate ligament reconstruction. Br J Sports Med 48(22):1613-1619

5. Ardern CL, Taylor NF, Feller JA, Whitehead TS, Webster KE (2013) Psychological responses matter in returning to preinjury 
level of sport after anterior cruciate ligament reconstruction surgery. Am J Sports Med 41(7):1549-1558

6. Ardern CL, Taylor NF, Feller JA, Whitehead TS, Webster KE (2015) Sports participation 2 years after anterior cruciate ligament reconstruction in athletes who had not returned to sport at 1 year: a prospective follow-up of physical function and psychological factors in 122 athletes. Am J Sports Med 43(4):848-856

7. Ardern CL, Webster KE, Taylor NF, Feller JA (2011) Return to sport following anterior cruciate ligament reconstruction surgery: a systematic review and meta-analysis of the state of play. Br J Sports Med 45(7):596-606

8. Barber-Westin SD, Noyes FR (2011) Factors used to determine return to unrestricted sports activities after anterior cruciate ligament reconstruction. Arthroscopy 27(12):1697-1705

9. Bates NA, Myer GD, Shearn JT, Hewett TE (2015) Anterior cruciate ligament biomechanics during robotic and mechanical simulations of physiologic and clinical motion tasks: a systematic review and meta-analysis. Clin Biomech 30(1):1-13

10. Brewer BW, Van Raalte JL, Cornelius AE, Petitpas AJ, Sklar JH, Pohlman MH, Krushell RJ, Ditmar TD (2000) Psychological factors, rehabilitation adherence, and rehabilitation outcome after anterior cruciate ligament reconstruction. Rehabil Psychol 45(1):20-37

11. Cherkin DC, Deyo RA, Street JH, Barlow W (1996) Predicting poor outcomes for back pain seen in primary care using patients' own criteria. Spine (Philadelphia Pa 1976) 21(24):2900-2907

12. Christino MA, Fantry AJ, Vopat BG (2015) Psychological aspects of recovery following anterior cruciate ligament reconstruction. J Am Acad Orthop Surg 23(8):501-509

13. Culliton SE, Bryant DM, Overend TJ, MacDonald SJ, Chesworth BM (2012) The relationship between expectations and satisfaction in patients undergoing primary total knee arthroplasty. $\mathrm{J}$ Arthroplast 27(3):490-492

14. Czuppon S, Racette BA, Klein SE, Harris-Hayes M (2014) Variables associated with return to sport following anterior cruciate ligament reconstruction: a systematic review. Br J Sports Med 48(5):356-364

15. Ellman MB, Sherman SL, Forsythe B, LaPrade RF, Cole BJ, Bach BR Jr (2015) Return to play following anterior cruciate ligament reconstruction. J Am Acad Orthop Surg 23(5):283-296

16. Everhart JS, Best TM, Flanigan DC (2015) Psychological predictors of anterior cruciate ligament reconstruction outcomes: a systematic review. Knee Surg Sports Traumatol Arthrosc 23(3):752-762

17. Faltstrom A, Hagglund M, Kvist J (2015) Factors associated with playing football after anterior cruciate ligament reconstruction in female football players. Scand J Med Sci Sports. doi:10.1111/ sms 12588

18. Feller J, Webster KE (2013) Return to sport following anterior cruciate ligament reconstruction. Int Orthop 37(2):285-290

19. Feller JA, Webster KE (2003) A randomized comparison of patellar tendon and hamstring tendon anterior cruciate ligament reconstruction. Am J Sports Med 31(4):564-573

20. Feucht MJ, Cotic M, Saier T, Minzlaff P, Plath JE, Imhoff AB, Hinterwimmer S (2016) Patient expectations of primary and revision anterior cruciate ligament reconstruction. Knee Surg Sports Traumatol Arthrosc 24(1):201-207

21. Gobbi A, Francisco R (2006) Factors affecting return to sports after anterior cruciate ligament reconstruction with patellar tendon and hamstring graft: a prospective clinical investigation. Knee Surg Sports Traumatol Arthrosc 14(10):1021-1028

22. Harris JD, Abrams GD, Bach BR, Williams D, Heidloff D, BushJoseph CA, Verma NN, Forsythe B, Cole BJ (2014) Return to sport after ACL reconstruction. Orthopedics 37(2):e103-e108

23. Heijne A, Axelsson K, Werner S, Biguet G (2008) Rehabilitation and recovery after anterior cruciate ligament reconstruction: patients' experiences. Scand J Med Sci Sports 18(3):325-335
24. Irrgang JJ, Anderson AF, Boland AL, Harner CD, Kurosaka M, Neyret P, Richmond JC, Shelborne KD (2001) Development and validation of the international knee documentation committee subjective knee form. Am J Sports Med 29(5):600-613

25. Kocher MS, Steadman JR, Briggs K, Zurakowski D, Sterett WI, Hawkins RJ (2002) Determinants of patient satisfaction with outcome after anterior cruciate ligament reconstruction. J Bone Joint Surg Am 84-A(9):1560-1572

26. Kvist J, Ek A, Sporrstedt K, Good L (2005) Fear of re-injury: a hindrance for returning to sports after anterior cruciate ligament reconstruction. Knee Surg Sports Traumatol Arthrosc 13(5):393-397

27. Langford JL, Webster KE, Feller JA (2009) A prospective longitudinal study to assess psychological changes following anterior cruciate ligament reconstruction surgery. Br J Sports Med 43(5):377-381

28. Lentz TA, Zeppieri G Jr, George SZ, Tillman SM, Moser MW, Farmer KW, Chmielewski TL (2015) Comparison of physical impairment, functional, and psychosocial measures based on fear of reinjury/lack of confidence and return-to-sport status after ACL reconstruction. Am J Sports Med 43(2):345-353

29. Levy AR, Polman RC, Clough PJ (2008) Adherence to sport injury rehabilitation programs: an integrated psycho-social approach. Scand J Med Sci Sports 18(6):798-809

30. Mondloch MV, Cole DC, Frank JW (2001) Does how you do depend on how you think you'll do? A systematic review of the evidence for a relation between patients' recovery expectations and health outcomes. CMAJ 165(2):174-179

31. Morrey MA, Stuart MJ, Smith AM, Wiese-Bjornstal DM (1999) A longitudinal examination of athletes' emotional and cognitive responses to anterior cruciate ligament injury. Clin J Sport Med 9(2):63-69

32. Muller U, Kruger-Franke M, Schmidt M, Rosemeyer B (2015) Predictive parameters for return to pre-injury level of sport 6 months following anterior cruciate ligament reconstruction surgery. Knee Surg Sports Traumatol Arthrosc 23(12):3623-3631

33. Nagao M, Doi T, Saita Y, Kobayashi Y, Kubota M, Kaneko H, Takazawa Y, Ishijima M, Kurosawa H, Kaneko K, Nozawa M, Ikeda H, Kim SG (2015) A novel patient-reported outcome measure for anterior cruciate ligament injury: evaluating the reliability, validity, and responsiveness of Japanese anterior cruciate ligament questionnaire 25. Knee Surg Sports Traumatol Arthrosc. doi:10.1007/s00167-015-3595-7

34. Osterberg A, Kvist J, Dahlgren MA (2013) Ways of experiencing participation and factors affecting the activity level after nonreconstructed anterior cruciate ligament injury: a qualitative study. J Orthop Sports Phys Ther 43(3):172-183

35. Pizzari T, McBurney H, Nicholas T, Feller J (2002) Adherence to anterior cruciate ligament rehabilitation: a qualitative analysis. J Sport Rehabil 11:90-102

36. Schmale GA, Kweon C, Larson RV, Bompadre V (2014) High satisfaction yet decreased activity 4 years after transphyseal ACL reconstruction. Clin Orthop Relat Res 472(7):2168-2174

37. Scott CE, Howie CR, MacDonald D, Biant LC (2010) Predicting dissatisfaction following total knee replacement: a prospective study of 1217 patients. J Bone Joint Surg Br 92(9):1253-1258

38. Swirtun LR, Renstrom P (2008) Factors affecting outcome after anterior cruciate ligament injury: a prospective study with a sixyear follow-up. Scand J Med Sci Sports 18(3):318-324

39. te Wierike SC, van der Sluis A, van den Akker-Scheek I, Elferink-Gemser MT, Visscher C (2013) Psychosocial factors influencing the recovery of athletes with anterior cruciate ligament injury: a systematic review. Scand J Med Sci Sports 23(5):527-540

40. Thomee R, Kaplan Y, Kvist J, Myklebust G, Risberg MA, Theisen D, Tsepis E, Werner S, Wondrasch B, Witvrouw E (2011) 
Muscle strength and hop performance criteria prior to return to sports after ACL reconstruction. Knee Surg Sports Traumatol Arthrosc 19(11):1798-1805

41. Waljee J, McGlinn EP, Sears ED, Chung KC (2014) Patient expectations and patient-reported outcomes in surgery: a systematic review. Surgery 155(5):799-808

42. Webster KE, Feller JA, Lambros C (2008) Development and preliminary validation of a scale to measure the psychological impact of returning to sport following anterior cruciate ligament reconstruction surgery. Phys Ther Sport 9(1):9-15

43. Zaffagnini S, Grassi A, Serra M, Marcacci M (2015) Return to sport after ACL reconstruction: how, when and why? A narrative review of current evidence. Joints 3(1):25-30 